\title{
An Iterated Complex Matrix Approach for Simulation and Analysis of Diffusion MRI Processes
}

\author{
Hans Knutsson, Magnus Herberthson and Carl-Fredrik Westin
}

\section{Conference Publication}

\section{Tweet}

N.B.: When citing this work, cite the original article.

Original Publication:

Hans Knutsson, Magnus Herberthson and Carl-Fredrik Westin, An Iterated Complex Matrix Approach for Simulation and Analysis of Diffusion MRI Processes, MEDICAL IMAGE COMPUTING AND COMPUTER-ASSISTED INTERVENTION - MICCAI 2015, PT I, 2015, pp.61-68.

http://dx.doi.org/10.1007/978-3-319-24553-9_8

Copyright: www.springer.com

Postprint available at: Linköping University Electronic Press

http://urn.kb.se/resolve?urn=urn:nbn:se:liu:diva-124149

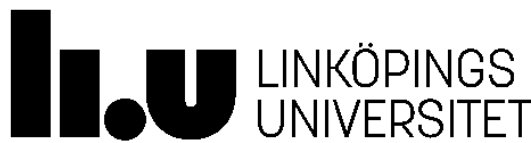




\title{
An Iterated Complex Matrix Approach for Simulation and Analysis of Diffusion MRI Processes
}

\author{
No Author Given \\ No Institute Given
}

\begin{abstract}
We present a novel approach to investigate the properties of diffusion weighted magnetic resonance imaging (dMRI). The process of restricted diffusion of spin particles in the presence of a magnetic field is simulated by an iterated complex matrix multiplication approach. The approach is based on first principles and provides a flexible, transparent and fast simulation tool. The experiments carried out reveals fundamental features of the dMRI process. A particularly interesting observation is that the induced speed of the local spatial spin angle rate of change is highly shift variant. Hence, the encoding basis functions are not the complex exponentials associated with the Fourier transform as commonly assumed. Thus, reconstructing the signal using the inverse Fourier transform leads to large compartment estimation errors, which is demonstrated in a number of $1 \mathrm{D}$ and $2 \mathrm{D}$ examples. In accordance with previous investigations the compartment size is under-estimated. More interestingly, however, we show that the estimated shape is likely to be far from the true shape using state of the art clinical MRI scanners.
\end{abstract}

\section{Introduction}

The field of diffusion weighted magnetic resonance imaging (dMRI) has developed rapidly over recent years and continues to attract a lot of attention. The dMRI observables can be taken as rough measures of tissue micro-structure, cell shape and general directions of the nerve fiber bundles. To move towards more precise measurements require a thorough understanding the process of restricted diffusion in the presence of a magnetic field gradient. To be able to correctly interpret the measurements attained by any given diffusion MR scan is of crucial importance. The commonly used $q$-space concept is equivalent to assuming a constant rate of spin angle phase change, i.e. constant local spatial frequency, across the compartment. A number of results exist showing that this concept holds an oversimplification of the process [1-6]. We present a novel simulation approach enabling a local spatial frequency analysis of the process which demonstrates that this is indeed the case. This fact and the intractability of analytic solutions have prompted a number of researcher to develop dMRI simulator tools [7-10]. A common feature of the simulators is, however, a boundary condition motivated, demodulation type, variable change, which is directly linked to the concept of $q$-space. Our approach is free from this type of a-priori modeling. 

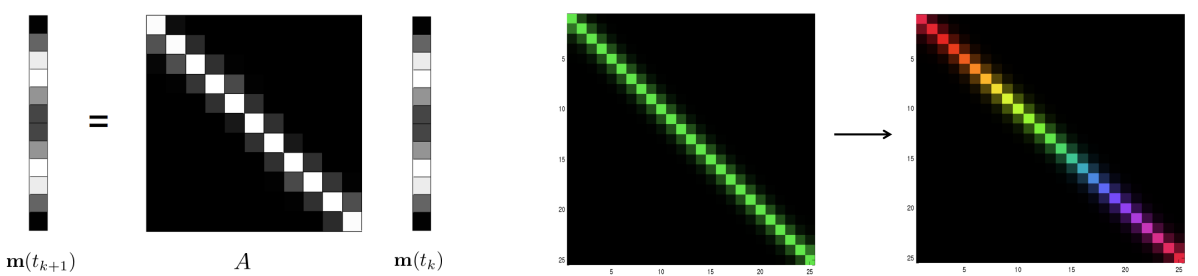

Fig. 1. Left - The process of diffusion can be formulated as an iterative matrix multiplication. Right - Color coded simulation matrices: A pure diffusion matrix is shown to the left. The matrix to the right shows how the strength of the local magnetic field increments the phase of the proton spins at each location. Zero phase is coded green, a phase of $\pi$ is coded red. Blue and yellow codes for $\pi / 2$ and $-\pi / 2$ respectively. The same color code is used in figures 2 and 4.

We believe the main advantages of our iterated complex matrix approach to be: First principles - Directly models local diffusion and spin phase change at all points, no additional boundary conditions needed. Flexibility - Any compartment shape can be easily specified as a function on a discrete grid and the gradient can be individually specified for each time step. Transparency - The development of the local magnetization can be monitored at all points and all times. Speed - A matlab simulation providing 10.000 measurements can typically be performed in less than a minute.

We have taken advantage of these features and continue to discuss some important consequences of the analysis carried out.

\section{Theory and Method}

Our method simulates the process of restricted diffusion of spin particles in the presence of a magnetic field by iterated matrix multiplications. To design the simulation matrix and carry out the experiments we used a nearest neighbor approach. The assumption made is that a quantized space-time can provide a good approximation of the continuous process. As illustrated in figure 1, the quantized diffusion process can be expressed as:

$$
\mathbf{m}\left(t_{k+1}\right)=A \mathbf{m}\left(t_{k}\right) .
$$

Here $\mathbf{m}$ is a complex vector of length $N$ holding the magnetization vectors for each unit. The simulation matrix, $A$, is an $N \times N$ complex matrix and $k$ is an integer indicating the diffusion time step number. This time step can be large, but initially $A$ is constructed for small time steps (see section 2.1) where $A$ then factors into two matrices: One matrix, $D$ simulating the diffusion process and one matrix, $G$, simulating the effect on the particle spin angles due to the applied magnetic field,

$$
A=G^{1 / 2} D G^{1 / 2}
$$

The diffusion matrix, $D$, is a real-valued matrix simulating only nearest neighbour interactions thus making it tri-diagonal in the 1-dimensional case. The 
construction is done in two steps: First the off-diagonal terms are set to specify the amount of particles that flow from the center unit to the neighbouring units. Conservation of mass requires that the columns of the resulting matrix are normalized to unity, i.e. $D$ is a left stochastic matrix. This implies that the columns will hold the probabilities for the new locations of the center unit particles after one time step. The rows specify the relative amount of particles in the adjacent units that move to the center unit. Thus, in general, $D$ will not be symmetric. The spin matrix, $G$, specifying the spin phase increment occurring during one time step, is a complex diagonal matrix: $G=\operatorname{diag}[\exp ((i \gamma g x \Delta t)]$, where $\gamma$ is the gyromagnetic ratio, $g$ is the applied magnetic gradient strength and $x$ is the spatial position of the corresponding unit.

\subsection{The short time limit, $\Delta t \rightarrow 0$}

Letting the time step become infinitely small by introducing a variable $n \rightarrow \infty$ we can express $A$ for finite times as:

$$
\begin{aligned}
A=A_{0}^{n}=\left(G_{0}^{1 / 2} D_{0} G_{0}^{1 / 2}\right)^{n} \quad \text { where } D_{0}=I+B \frac{\Delta t}{n} \\
\quad \text { and } \quad G_{0}=I+i \underbrace{\gamma g \operatorname{diag}\left[x_{1}, \ldots, x_{n}\right]}_{C} \frac{\Delta t}{n}
\end{aligned}
$$

Here $B$ is tri-diagonal, and $C$ is diagonal. Note that the diffusion mass conservation requirement of $D$ implies that the column sums of $B$ equal zero. Examining $A_{0}$, which depends on $n$, leads to:

$$
\begin{aligned}
A_{0} & =G_{0}^{1 / 2} D_{0} G_{0}^{1 / 2} \approx\left(I+\frac{i C \Delta t}{2 n}\right)\left(I+\frac{B \Delta t}{n}\right)\left(I+\frac{i C \Delta t}{2 n}\right) \\
& \approx I+(B+i C) \frac{\Delta t}{n}
\end{aligned}
$$

This shows that, for very short time steps, $\Delta t$, we arrive at the simple form of $A$ shown below (left). Further, by combining equations 1 and 4 in the limit $\Delta t \rightarrow 0$, the process can be expressed equivalently as the differential equation to the right below.

$$
A=e^{(B+i C) \Delta t} \quad \longleftrightarrow \quad \frac{\partial \mathbf{m}}{\partial t}=(B+i C) \mathbf{m}
$$

This highlights the fundamental structure of the process of diffusing spin particles in the presence of a magnetic field gradient. The differential equation above can, in fact, be seen as equivalent to the Bloch-Torre equation, [1], on a discrete grid, which is an indication of the soundness of our matrix simulation approach.

\subsection{From compartment shape to simulation matrix}

A major advantage over traditional simulation approaches is that our simulation matrix approach allows compartments of any shape to be specified. The compartment is specified as a function on a grid in any dimension and the matrix 

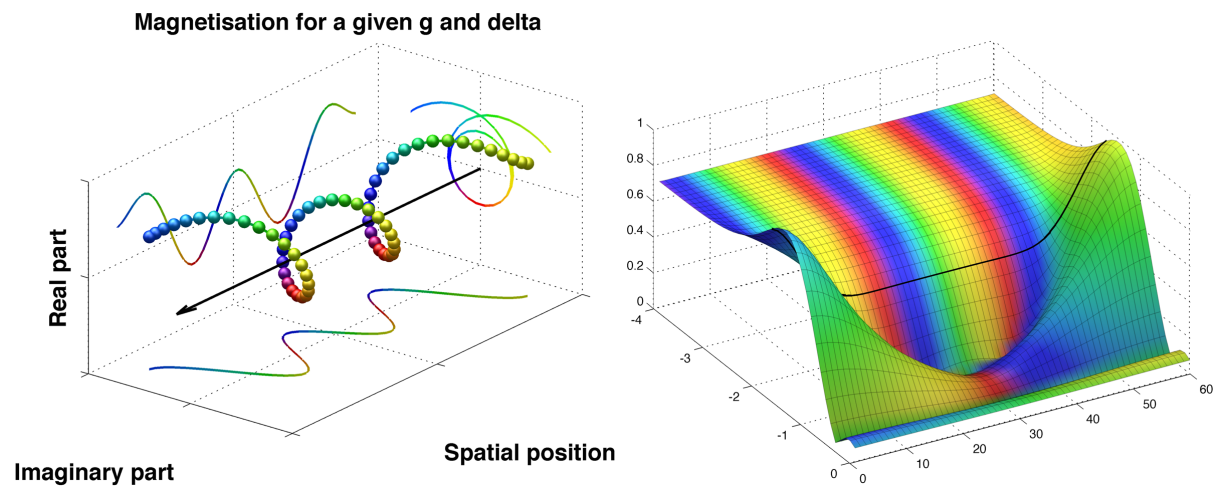

Fig. 2. Left - Positions in the complex plane of 60 magnetization vectors across the compartment for one gradient strength and $\delta=0.05 \Delta$. Colors indicate phase angle. The real part is plotted on the back plane and the imaginary part on the bottom plane. The same color code as in figure 1 is used for both plots. Right - Magnetization across the compartment (positions 1 to 60 ) for $\delta$ from $10^{-4} \Delta$ to $1.0 \Delta$ (log10 scale). Z-axis shows magnitude and color shows phase. The black line marks $\delta=0.05 \Delta$.

components can then be directly found. Work aiming in a similar direction can be found in $[9,10]$. In the $1 \mathrm{D}$ experiments presented here the compartment was quantized to consist of 60 adjacent units. The compartment sizes were found by equidistant sampling of a specified continuous compartment function. The interface sizes were found as the harmonic mean of the flanking compartment sizes. The off diagonal terms in $\mathrm{D}$ were set to be proportional to the interface size.

The principles behind the simulation matrix design are readily generalized to higher dimensions. In 2 dimensions we use 4-connectivity and both the diffusion matrix, $D$, and the spin matrix, $G$, become block matrices. As before $D$ hold the probabilities of a particle moving from the center unit to an adjacent unit but is now a block tri-diagonal matrix. Further, the gradient, $g$, and the spatial position, $x$, become vectors, $\mathbf{g}$ and $\mathbf{x}$. Thus, in $2 \mathrm{D}$, the components of the spin matrix will be given by:

$$
G=\operatorname{blockdiag}\left[e^{i \gamma \mathbf{g}^{T} \mathbf{x} \Delta t}\right]
$$

\section{Results}

The results presented below are from simulations in one and two dimension. The method is, however, equally applicable in three (and higher) dimensions. Results on local frequency are for visualization purposes shown using 1-dimensional compartments. The simulations studying compartment estimation are carried out for 2-dimensional compartments.

Simulated sequences - The sequences simulated in the experiments were the standard single PFG sequences with encoding parameters $\delta$ and $\Delta$ and gradient strength $g$. The length of $\delta$ was varied logarithmically from $10^{-4} \Delta$ to $1 \Delta$, 

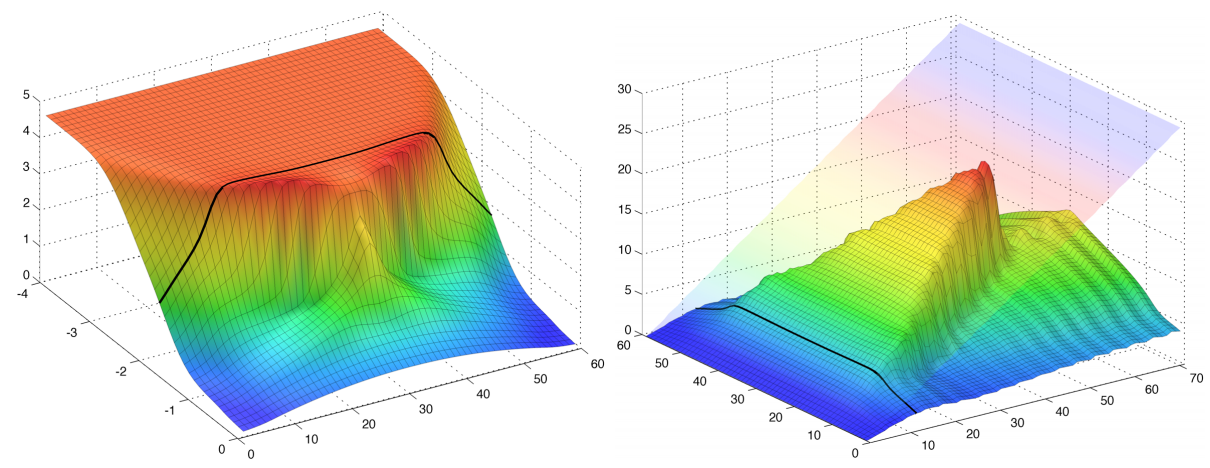

Fig. 3. Left - Color and height shows the local spatial frequency of the magnetization for a given gradient strength. $\mathrm{x}$ - and $\mathrm{y}$-axis same as in figure 2 right. The black line marks $\delta=0.05 \Delta$. Right - Color and height shows the local frequency for different positions across the compartment for $\delta=0.05 \Delta$. Spatial position (1-60) is indicated on the $\mathrm{x}$-axis. The $\mathrm{y}$ - axis indicates the applied gradient strength. The black lines mark the $g$ used in figure 2 .

corresponding to the number of time steps, $K$, ranging from 50 to 500000 . The simulation time for 2000 instances $(40 \delta, 50 \mathrm{~g})$ was 13 seconds running matlab on a standard portable computer.

Local spatial frequency - To analyze the diffusion process, the local spatial frequency of the magnetization $\omega(x)$ was computed. The definition of local frequency is given by:

$$
\omega(x)=\frac{\partial \arg (\mathbf{m})}{\partial(x)}
$$

Figure 2 (left) visualizes compartment magnetization for $g$ corresponding to 2.60 cycles across the compartment using the short pulse approximation (SPA). SPA predicts a complex exponential magnetization across the compartment, i.e. constant local spatial frequency. Even though $\delta$ is very short the simulated magnetization clearly deviates from the perfect spiral of a complex exponential and the total number of cycles is only 2.20. Figure 2 (right) shows the compartment magnetization when varying $\delta$. The well known edge effects [2-5] are noticeable already for $\delta=10^{-3} \Delta$ and increase to be extreme roughly at $\delta=0.1 \Delta$. For longer $\delta$ the averaging effect of the diffusion effectively prevents a build up of a strong local magnetization. The black line indicates the location of $\delta=0.05 \Delta$, the value used to render the left plot.

Figure 3 (left) shows the local frequency dependency on $\delta$ for the same $g$ as in figure 2. For lengths up to $\delta=10^{-3} \Delta$ the SPA is valid and the local frequency constant across the compartment. For longer $\delta$ the local frequency consistently decreases as the position approaches the compartment edges. The distance from the edge at which the decrease begins increases with increasing $\delta$ and at $\delta=0.1 \Delta$ 
this edge effect reaches all the way to the center of the compartment. For longer $\delta$ the local frequency is everywhere much lower than predicted by the SPA. Figure 3 (right) shows the local frequency dependence on $g$ for $\delta=0.05 \Delta$. For reference the transparent plane shows the SPA prediction. As $g$ increases the local frequency drop gets more pronounced and the effect spreads toward the compartment center. At a $g$ corresponding to 7 cycles across the compartment the edge effect reaches all the way to the center. The black lines in figure 3 indicate the location of $\delta=0.05 \Delta$, thus showing the same function but in different contexts.

Simplistically summarized - Using clinical scanners, high $b$-values do not lead to high $q$-values.

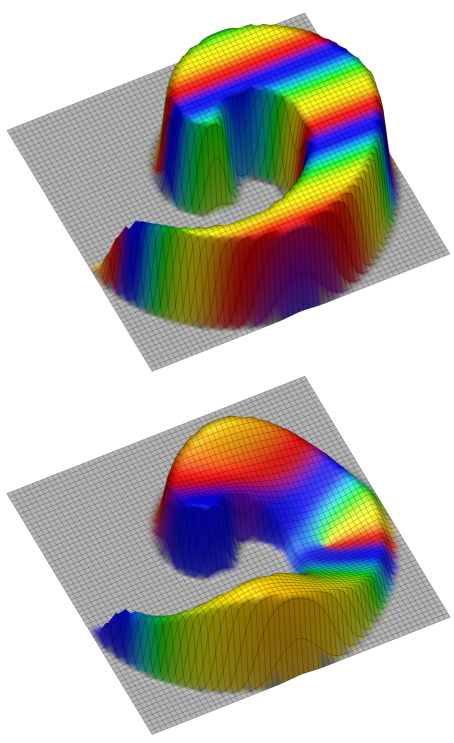

Magnetization and geometry - To illustrate the generality of our approach and the interplay between geometry and local magnetization during a dMRI scan a more elaborate 2-dimensional compartment was designed. Figure 4 shows the local magnetization for a spiral compartment at one measurement point in $q$-space. The lower plot clearly demonstrates that the encoding basis functions are far from the top plot complex exponentials commonly assumed. It is clear that the basis created will be dependent on the geometry of each individual compartment present in one voxel. Thus, to quote a giant in the field, '...the concept of $q$-space no longer has any meaning...' [1] p356.

\subsection{Compartment estimation}

In this section we present results showing how a Fourier transform based estimation of the spatial compartment shape will appear for in a number of different situations. The results presented cor-

Fig. 4. A spiral compartment demonstrating the effect of geometry on local magnetization for one measurement point in qspace. The magnetization magnitude is given by the height and color indicates phase (the same color code as in figures 1 and 2). The ideal case is shown on top, the lower plot shows a realistic case.

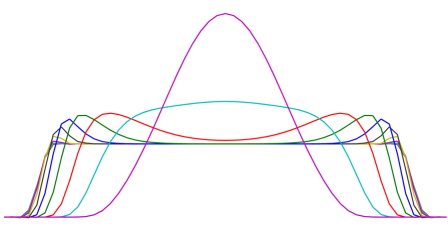

Fig. 5. Reconstructions in 1dimension using the 'short-long' single PFG sequence: The different curves represent reconstructions when $\delta$ is varied logarithmically over a range of $10^{5}$ keeping the product $g \delta$ constant. respond to the use of a sPFG sequence consisting of a first gradient, $\mathrm{g}$, applied during a time $\delta$ followed by a very weak gradient, $\mathbf{g}_{l}$, of the opposite sign during a long time, $\delta_{l}$, where $\mathbf{g}_{l} \delta_{l}=-\mathbf{g} \delta$. This type of 'short-long' sPFG pulses will lead to that the measurement attained give the Fourier transform of the compartment [6]. Hence deviations can be studied by making 'reconstructions' using an inverse Fourier transform. 
Compartment estimation in one dimension - To show the general features of the dMRI measurement protocol 1D simulations followed by an inverse Fourier transform were performed. Figure 5 shows the reults when varying $\delta$ logarithmically over a range of $10^{5}$ keeping the q-values, i.e the products $g \delta$, identical. The results are consistent with the findings studying local frequency and show that very short delta, $\delta=10^{-3} \Delta$ or shorter, are needed for a good reconstruction. At $\delta=510^{-3} \Delta$ (the black curve) the edge effect is clearly visible and at $\delta=0.3 \Delta$ (the cyan curve) the reconstruction distortion is severe.

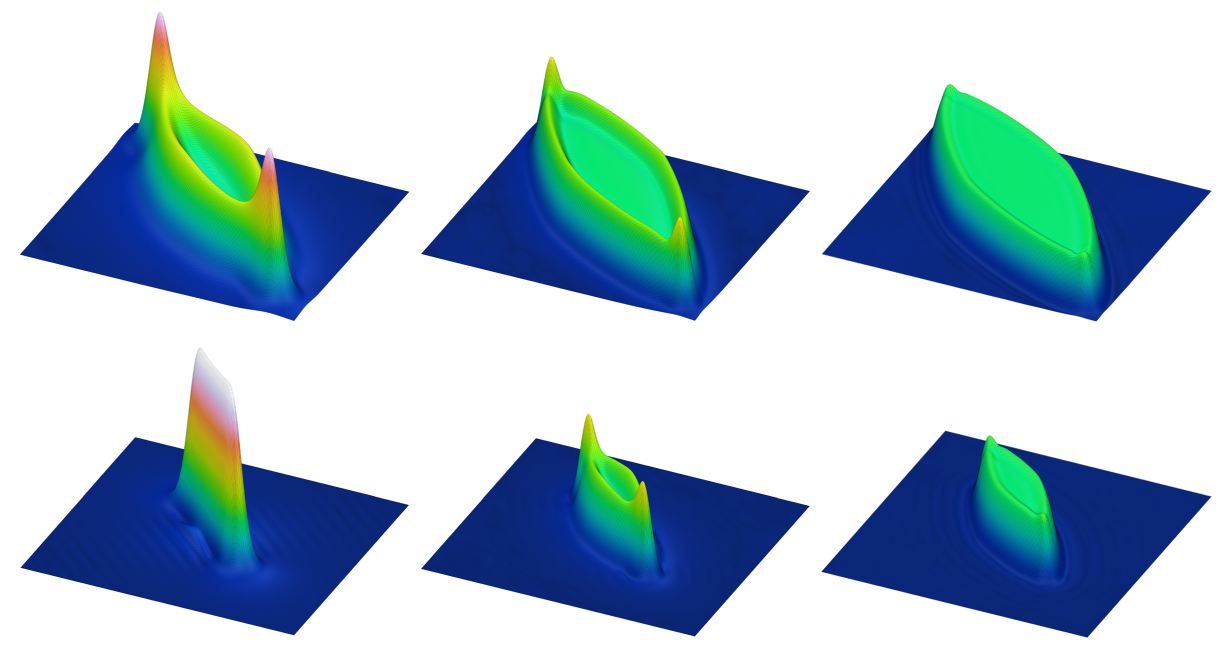

Fig. 6. 2D reconstruction results for two different compartment sizes and three different scanner settings. Top row compartment size $=14 \times 40 \mu \mathrm{m}$. Bottom row compartment size $=7 \times 20 \mu \mathrm{m}$. Parameters from left to wright: (1) $\delta_{\text {Max }}=80 \mathrm{mS}, \mathrm{g}=150 \mathrm{mT} / \mathrm{m}$, (2) $\delta_{\text {Max }}=8 \mathrm{mS}, g=1500 \mathrm{mT} / \mathrm{m}$, (3) $\delta_{\text {Max }}=0.8 \mathrm{mS}, g=15 \mathrm{~T} / \mathrm{m}$,

Compartment estimation in two dimensions - Results from different 2D reconstructions of two compartments having the same shape but different size are shown in figure 6 . The leftmost plots show results using parameters corresponding to a good MR scanner. The middle and right plots show the results if 10 (middle) or 100 (right) times higher gradients were available. It is clear that the distorting 'edge effects' seen in 1D become much more complex in 2D and here also radically changes the apparent shape of the compartment. Only at the unrealistic $g$-value of $15 \mathrm{~T} / \mathrm{m}$ are the compartment reconstructions good replicas of the true shapes. Interestingly, the distortions clearly increases compartment anisotropy.

\section{Conclusion and Discussion}

We have presented a novel simulation tool to study diffusion weighted magnetic resonance imaging (dMRI). The flexibility, transparency and speed of our iterated matrix approach has allowed us to investigate a number of important 
properties of dMRI. We have shown that there is a significant decrease of the local spatial frequency of the magnetization at the compartment at clinical values of $\delta$ and that this will have a major impact on compartment size and shape estimates using traditional modeling. However, diffusion MRI scans will continue to hold a lot of information and following the leads presented above we feel confident that better models for extracting micro structure properties will be put forward. The power of these models can then be tested using simulations and, when found to be improvements on state of the art, find their way into clinical practise.

\section{Acknowledgement}

Intentionally left blank for the blind review process.

\section{References}

1. Callaghan, P.T.: Principles of nuclear magnetic resonance microscopy. Volume 3. Clarendon Press Oxford (1991)

2. Mitra, P., Halperin, B.: Effects of finite gradient-pulse widths in pulsed-fieldgradient diffusion measurements. JMR, Series A 113(1) (1995) $94-101$

3. Stepisnik, J., Duh, A., Mohoric, A., Sersa, I.: MRI edge enhancement as a diffusive discord of spin phase structure. JMR (San Diego, Calif : 1997) 137(1) (1999) 154160

4. Åslund, I., Cabaleiro-Lago, C., Söderman, O., Topgaard, D.: Diffusion NMR for determining the homogeneous length-scale in lamellar phases. The journal of physical chemistry. B 112(10) (2008) 2782-2794

5. Åslund, I., Topgaard, D.: Determination of the self-diffusion coefficient of intracellular water using PGSE NMR with variable gradient pulse length. JMR (San Diego, Calif : 1997) 201(2) (2009) 250-254

6. Laun, F.B., Kuder, T.A., Wetscherek, A., Stieltjes, B., Semmler, W.: NMR-based diffusion pore imaging. Physical Review E 86(2) (2012) 021906

7. Drobnjak, I., Siow, B., Alexander, D.C.: Optimizing gradient waveforms for microstructure sensitivity in diffusion-weighted MR. Journal of Magnetic Resonance 206(1) (2010) 41-51

8. Russell, G., Harkins, K.D., Secomb, T.W., Galons, J.P., Trouard, T.P.: A finite difference method with periodic boundary conditions for simulations of diffusionweighted magnetic resonance experiments in tissue. Physics in Medicine and Biology $\mathbf{5 7}(4)(2012) \mathrm{N} 35$

9. Nguyen, D.V., Li, J.R., Grebenkov, D., Bihan, D.L.: A finite elements method to solve the Bloch-Torrey equation applied to diffusion magnetic resonance imaging. Journal of Computational Physics 263(0) (2014) 283 - 302

10. Li, J.R., Calhoun, D., Poupon, C., Le Bihan, D.: Numerical simulation of diffusion MRI signals using an adaptive time-stepping method. Physics in medicine and biology 59(2) (2014) 441 